\title{
Two-Year Treatment Tracking of Dermatomyositis Patient Complicated With Massive Pericardial Effusion and Stubborn Dysphagia: A Case Report and Literature Review
}

\author{
Yi Bao ${ }^{\mathrm{a}, \mathrm{c}}$, Dianyuzi Xie ${ }^{\mathrm{b}}$, Miao Zhang ${ }^{\mathrm{a}}$, Xingyue $\mathrm{Li}^{\mathrm{b}}$, Xinyu Du ${ }^{\mathrm{b}}$, \\ Sijing Li ${ }^{b}$, Yayong Ding ${ }^{b}$, Fen Qiu $^{\mathrm{b}}$
}

\begin{abstract}
Dermatomyositis is a connective tissue disease mainly involving striated muscles and skins. It is rare to complicate with massive pericardial effusions. In this article, we tracked a 2-year treatment history of a dermatomyositis patient with rare complications. In the late stage of the disease, massive pericardial effusion was combined. Complications such as dysphagia, pulmonary hypertension, Sjogren's syndrome and anticardiolipin antibody syndrome posed great challenges to treatment. Massive pericardial effusion and pulmonary failure resulted in death of the patient, and anticardiolipin antibody syndrome was also one of the possible important causes of death. Good compliance could help control the disease. Massive pericardial effusion should be punctured in time. When combined with dysphagia, adequate intravenous immune globulin (IVIG) was the key to treatment.
\end{abstract}

Keywords: Dermatomyositis; Pericardial effusion; Dysphagia; IVIG

\section{Introduction}

Dermatomyositis is a connective tissue disease mainly involving striated muscles and skins [1-3]. Cardiac involvement is common, manifested as arrhythmia, such as palpitations and arrhythmias. It is rare to complicate with massive pericardial effusions.

We encountered a patient with dermatomyositis complicated with massive pericardial effusion, However, in the late stage of the disease, the patient developed stubborn dysphagia, pulmonary hypertension, Sjogren's syndrome (SS) and anticardiolipin antibody syndrome, which posed great challenges

Manuscript submitted October 25, 2018, accepted November 12, 2018

aDepartment of Neurology, Taihe Hospital Affiliated to Hubei University of Medicine, Shiyan City, Hubei Province, China

${ }^{b}$ Hubei University of Medicine, Shiyan City, Hubei Province, China

${ }^{\mathrm{c} C}$ Corresponding Author: Yi Bao, Department of Neurology, Taihe Hospital Affiliated to Hubei University of Medicine, Shiyan City, Hubei Province, China. Email: karlbaoyi@163.com

doi: https://doi.org/10.14740/jmc3202 to the treatment. In this paper, the experience of treating this patient for 2 years after onset is reported as follows.

\section{Case Report}

The patient is a 47 -year-old female of $152 \mathrm{~cm}$ in height and 45 $\mathrm{kg}$ in weight, with no special medical history.

On May 31, 2016, she was admitted to hospital due to "double lower limbs pain $>20$ days". She was diagnosed as polymyositis and discharged after treatment.

On July 19, 2016, due to "double lower limb pain, weakness for 3 months and diarrhea for 2 days", she was admitted to hospital. The results of diagnosis were dermatomyositis, pulmonary infection and sinus tachycardia. She was given hormone and symptomatic supportive treatment. During the course of the disease, the patient developed wheezing, coughing and swallowing weakness, and the muscle strength of the limbs decreased significantly. After intravenous immune globulin (IVIG), she was discharged after hospitalization, taking regular prednisone and other drugs, and was reviewed regularly.

On July 11, 2017, due to "the rash on the left lower extremity and pain > 10 days", she was admitted to the hospital. The results of diagnosis were herpes zoster neuralgia and mixed connective tissue disease. She was given antivirus, immunosuppression (methotrexate, prednisone, hydroxychloroquine) and other treatments. After the symptoms were improved, she was discharged and told to take medicine regularly outside the hospital. However, the patient's self-perception symptoms were significantly improved, and she stopped taking medicine by herself and return to work, with no regular follow-up visit. When there were cases such as chest tightness and myalgia, she did not go to the doctor in time.

On July 7, 2018, due to "chest suffocation with dizziness > 10 days and diarrhea for 2 days" she was admitted to the hospital. The results of physical examination were: temperature $37.1^{\circ} \mathrm{C}$, pulse 107 beats $/ \mathrm{min}$, respiratory rate 28 beats $/ \mathrm{min}$ and blood pressure $110 / 80 \mathrm{~mm} \mathrm{Hg}$. There were symptoms of acute facial features, facial erythema, jugular vein anger, multiple axillary lymph node enlargement, lung dampness, flask heart, distant heart, pulmonary artery systolic ejection murmurs, systolic reflux murmurs in tricuspid regurgitation, left lower abdominal tenderness, mild edema in both lower extremities 
Table 1. Comparison of Related Indicators Before and After Treatment in 2 years

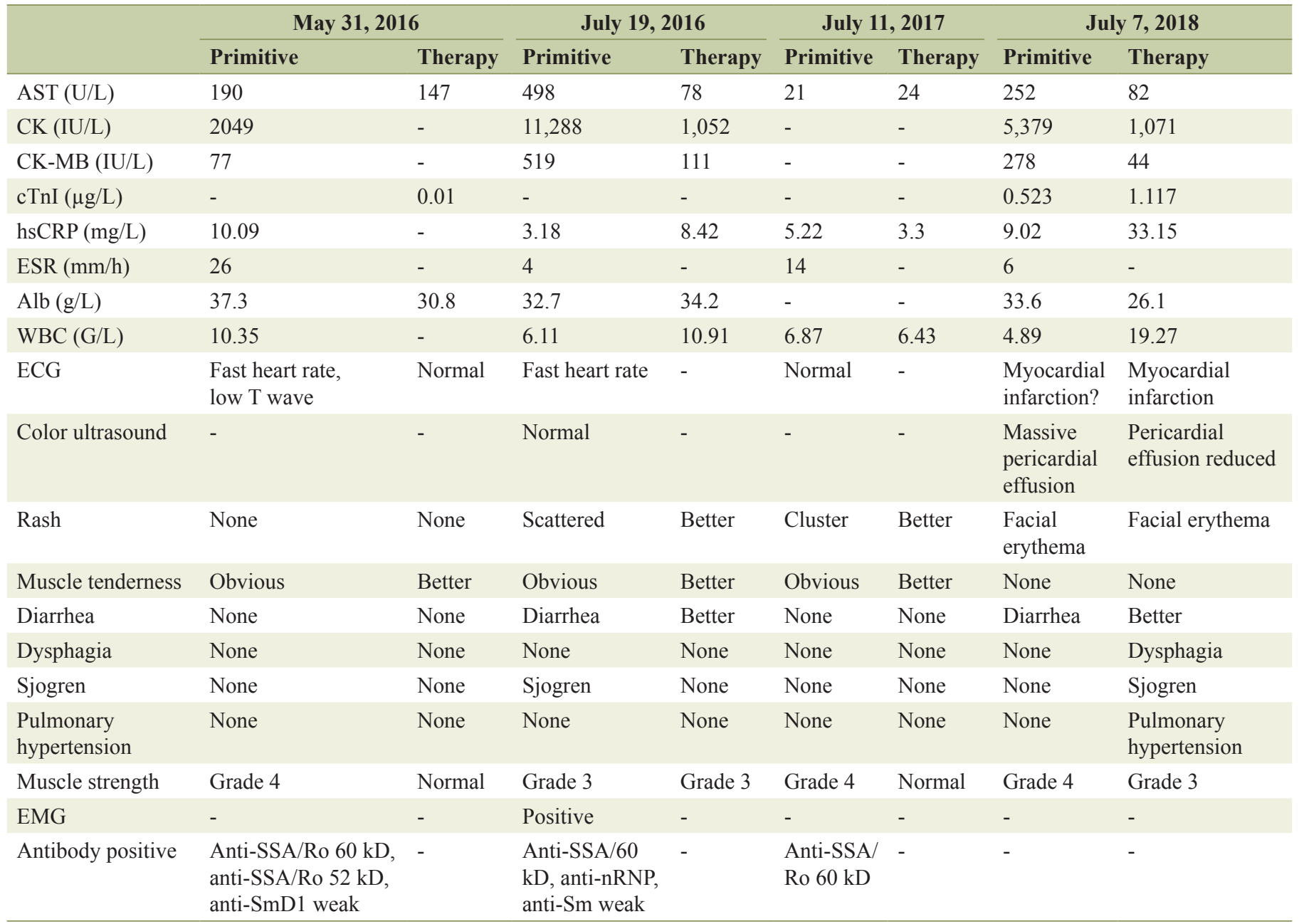

AST: aspartate aminotransferase; CK: creatine kinase; cTnl: cardiac troponin I; CPR: cardio pulmonary resuscitation; ESR: equivalent series resistance; Alb: albumin; WBC: white blood cell; ECG: electrocardiograph; EMG: electromyography.

and level 4 proximal muscle strength. The results of diagnosis were massive pericardial effusion, acute myocardial infarction, dermatomyositis, pulmonary infection and hypoalbuminemia. Dermatomyositis was considered to invade the heart and cause massive pericardial effusion. She was given methylprednisolone $(500 \mathrm{mg})$ shock and IVIG (5 g) to regulate immunity, and symptomatic supportive treatment, and departments of endocrinology, cardiology, cardiothoracic surgery and respiratory were asked to assist in the diagnosis and treatment. Pericardial puncture was recommended for the patient, but she disagreed. She underwent dynamic review of cardiac color Doppler ultrasound, electrocardiogram, tests for myocardial enzyme, troponin, B-type natriuretic peptide (BNP) and electrolyte levels, and close observation of changes in the condition.

On July 15, 2018, the patient had obvious limb edema, dyspnea and hernias were significantly aggravated, and she was treated with diuretic detumescence.

On July 21, 2018, edema of the limbs was significantly subsided, but symptoms such as hoarseness, difficulty in articulation, dryness and difficulty in swallowing were present.
When water or liquid food was ingested, it flowed out through the nostrils, causing cough, but the sputum was difficult to cough up. Dewatering drugs were discontinued and the upper gastric tube was given, and fluconazole antifungal treatment was added for the oral mucosa.

On July 24, 2018, the patient's vital signs were not stable. After using norepinephrine, the blood pressure can be maintained at $80 / 40 \mathrm{~mm} \mathrm{Hg}$, the heart rate was 160 beats/min and the breathing was 40 beats $/ \mathrm{min}$. She was advised to take a central venous catheter to monitor the central venous pressure and input plasma support treatment. After the patient and her relatives were informed about the condition, they requested to transfer out of the intensive care unit (ICU).

On July 26, 2018, 11:56 a.m., the patient showed a progressive decline in heart rate, respiratory slowdown and dilated pupils, blood pressure cannot be measured, limbs were wet and cold. She was immediately given cardiopulmonary resuscitation (CPR) and intravenous injection of epinephrine. After half an hour of continuous rescue, the patient still had no spontaneous heartbeat recovery, and her family members 


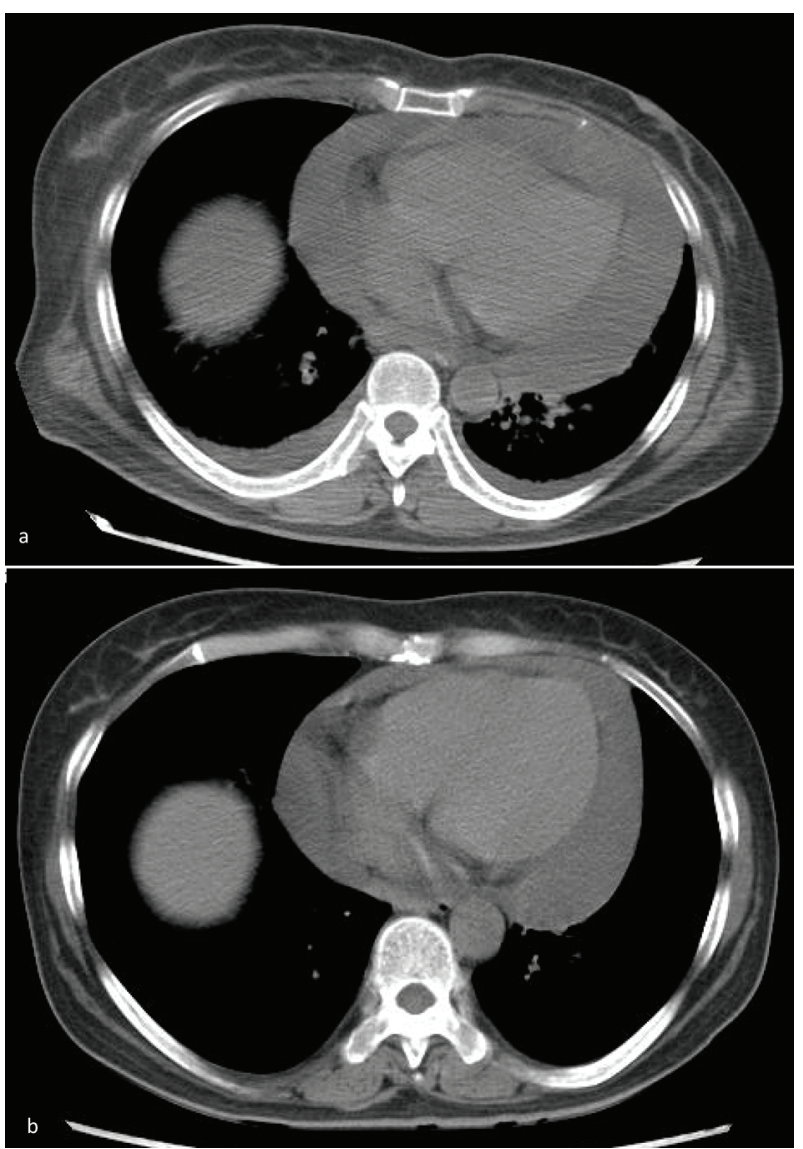

Figure 1. (a) On July 7, 2018, on admission, chest CT showed massive pericardial effusion. (b) On July 23, 2018, after treatment, pericardial effusion decreased significantly.

requested to give up the rescue and signed.

Two years of specific medical tracking history included: symptoms, signs, imaging examinations, laboratory tests, and treatment plans and outcomes (Table 1 and Supplementary Table 1). A large number of pericardial effusion changes were shown in Figures 1 and 2.

\section{Discussion}

This patient had symmetrical proximal muscle weakness, pain, dysphagia and dyspnea, characteristic skin lesions, increased serum creatine kinase (CK), CK isoenzyme (CK-MB), lactate dehydrogenase (LDH), aspartate aminotransferase (AST), alanine aminotransferase (ALT) and related antibodies, and electromyography (EMG) and positron emission tomographycomputed tomography (PET-CT) suggested myositis. According to the diagnostic criteria proposed by Bohan and Peter in 1975 [1], dermatomyositis can be diagnosed.

The patient's symptoms were repeated and the heart was invaded within 2 years. At the last hospital admission, there were massive pericardial effusion and respiratory distress, and the symptoms were improved after treatment with hormones, immunosuppressants and IVIG, but in the process of treat- ment, the occurrence of stubborn dysphagia, pulmonary hypertension and SS, leading to respiratory and circulatory failure, posed great challenges to treatment.

Causes of treatment failure were as follows: 1) dermatomyositis is easy to recurrent and aggravate; 2 ) in the second hospitalization, the patient was in critical condition and discharged without cured; 3) after discharge for the third time, the patient stopped the medicine too early. When she had chest tightness and discomfort, she did not go to the hospital in time; 4) not timely pericardial pumping, heart compression for too long time, leading to circulatory failure; 5) not enough IVIG; 6 ) persistent dysphagia and insufficient intake lead to hypovolemia; 7) high fever, considered as isotonic water loss, failed to be corrected in time; 8) progressive dyspnea, leading to respiratory failure; 9) progressive reduction of platelets, combined anticardiolipin antibody syndrome was also needed to considered; 10) electrocardiogram indicated extensive anterior wall myocardial infarction, which was also an important cause of death.

Deficiencies were: 1) no pictures of skin damage; 2) no muscle biopsy, no pathological data; 3) lack of data such as complement, uric acid, myoglobin, etc.; 4) inadequate disclosure of the disease condition and poor patient compliance; 5) failing to persuade her husband to complete pericardial puncture and aspiration in time; 6) failing to solve the patient's dysphagia in time; 7) when dysphagia combined with SS was present, failing to convince the family to use sufficient amount IVIG in time.

Dermatomyositis is an autoimmune disease that damages the skins and muscles. Involvement of proximal muscles of both lower limbs is most common, presenting as standing difficulty and gait limbus. When the muscles of pharynx, upper esophagus and palate are involved, hoarseness and dysphagia may occur. When the diaphragm and intercostal muscles are involved, dyspnea, dyspnea and pulmonary infection may occur. Myocardial involvement can lead to heart failure [1-3].

Numerous studies have shown that glucocorticoids are the first choice for treating dermatomyositis. However, in severe cases, the early application of immunosuppressive agents (methotrexate or azathioprine) combined with glucocorticoid can significantly improve muscle strength and reduce the dosage of hormones. In addition, cyclophosphamide, leflunomide, low-dose cyclosporin A, antimalarials, immunoglobulins, etc., can all play a role in refractory dermatomyositis. The disease is chronic. The treatment effect depends on the type of disease, treatment plan, patient and the degree of patient and family cooperation. After the condition is stable, the dosage can be reduced appropriately and maintained for a long time. If the drug is stopped prematurely, recurrence can be caused [4-6]. Galimberti et al [7] treated 42 patients with refractory dermatomyositis with IVIG. The results showed that $83 \%$ of the patients were improved, and the exposure of glucocorticoid and immunosuppressive drugs was reduced.

Cardiac involvement is common and generally mild. Studies by Guerra et al have found that up to $50 \%$ of patients with inflammatory myopathy have clinical heart disease, presenting with increased CK-MB and myoglobin, but this is not necessarily related to myocardial involvement, most of which may be generated by damaged muscles [8]. Prior to 2005, it was 
The trend chart of pericardial effusion

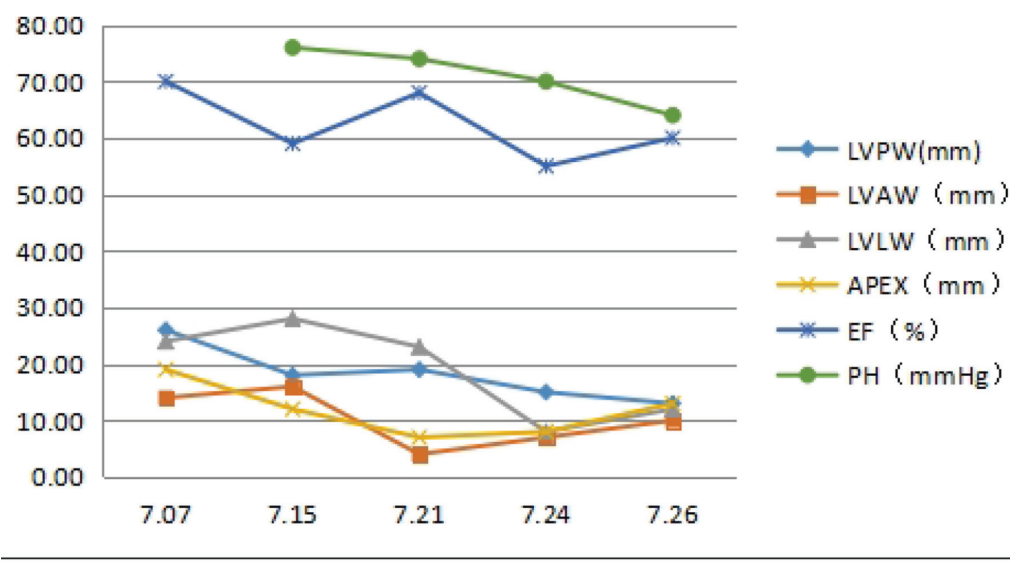

The trend chart of pericardial effusion related indicators

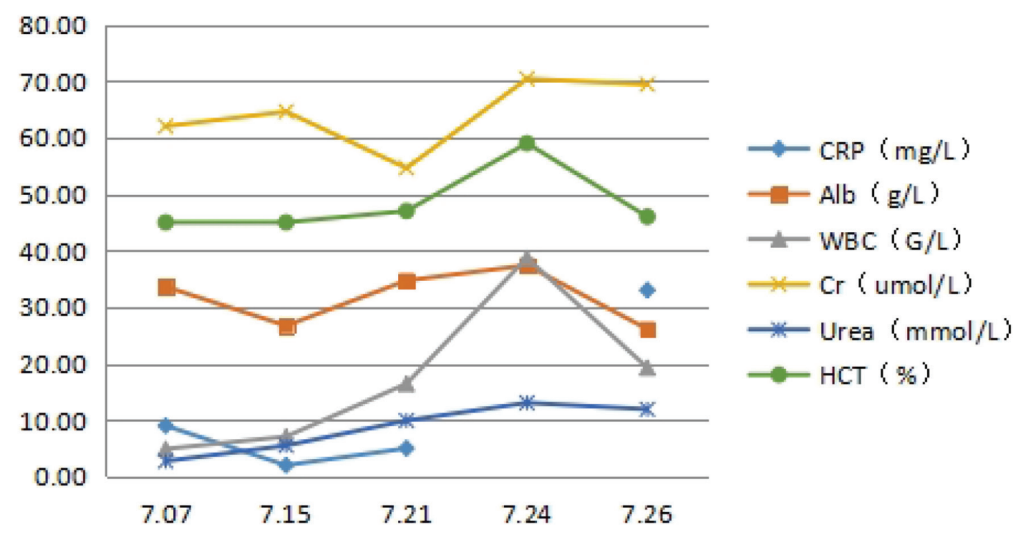

Figure 2. Trends in pericardial effusion and various indicators during treatment. Patients with a large amount of pericardial effusion, with the treatment gradually reduced, but in the late stage of the disease, secondary to difficult dysphagia, the relevant indicators change significantly, especially at 7.24. White blood cells, hematocrit and creatinine increased significantly, respiratory rate and blood pressure increased, and oxygen saturation decreased, posing a significant challenge to treatment, due to severe perfusion, suggesting that IVIG should be added promptly when patients have difficulty in swallowing. LVPW: left ventricular posterior wall; LVAW: anterior left ventricular wall; LVLW: left ventriculer lateral wall; EF: ejection fraction; PH: pulmonary hypertension; CPR: cardiopulmonary resuscitation; Alb: albumin; WBC: white blood cell; Cr: creatinine; HCT: hematocrit.

generally believed that pericardial involvement was rare, but Kamath et al reported that it was not uncommon to find pericardial involvement through autopsy [4].

Dermatomyositis can cause pericardial effusion, and massive pericardial effusion is a life-threatening event that requires rapid diagnosis and immediate intervention to prevent fatal outcomes. Emergency pericardial puncture and drainage under the guidance of color Doppler ultrasound is usually adopted to reduce the mortality rate. In severe cases, indwelling catheter drainage is required, and percutaneous balloon pericardial incision is also used for emergency treatment [9]. Also some studies have shown that extracting the pericardial effusion is not applicable to all patients. Napp et al [10] reported a risk of pericardial decompression syndrome in patients with 4,050 $\mathrm{mL}$ pericardial effusion. Tanizaki et al [11] reported that for blood, non-surgical treatment may be a viable treatment option for patients with massive pericardial effusions with unstable flow kinetics.
Dysphagia is a potentially life-threatening complication of dermatomyositis and may be present in up to $60 \%$ of cases. However, a sudden onset of dysphagia without significant exacerbation of other symptoms can make diagnosis and treatment challenging $[12,13]$. De et al [14] showed that in the treatment of dermatomyositis with corticosteroids and immunosuppressants, $70 \%$ of patients were prone to dysphagia, and autopsy showed that most of the causes were distal esophageal motility disorders. Ertekin et al [15] evaluated pharyngeal swallowing by electrophysiological methods, and the finding demonstrated the weakness of the striated oropharyngeal muscles. Cricopharyngeal sphincter muscle was affected less frequently. Kwon et al [16] treated a 53-year-old male patient with sudden dysphagia (methylprednisolone, azathioprine and IVIG). The patient could swallow saliva at 2 months and returned to normal at 3 months. Paez et al [12] also reported that severe dysphagia occurred during the treatment of dermatomyositis with corticosteroids and methotrexate, and one patient 
improved after IVIG. Deepak et al [17] successfully treated a 46-year-old female patient with dysphagia by IVIG.

Most dermatomyositis can be secondary to interstitial pneumonia, and pulmonary function tests and lung biopsy can confirm the diagnosis. Minai et al [18] reported three cases of pulmonary hypertension secondary to dermatomyositis in 2009 , and described related clinical symptoms and therapeutic responses. Patients with pulmonary fibrosis were often accompanied by chronic hypoxia and carbon dioxide retention, resulting in extensive narrowing of the pulmonary arterioles and increased pulmonary circulation resistance, leading to pulmonary hypertension. Symptoms included dyspnea, fatigue, fatigue, reduced exercise tolerance, syncope, angina or chest pain, hemoptysis and hoarseness. Glucocorticoid remains the preferred drug, followed by immunosuppressive agents [1921].

SS is a chronic autoimmune disease mainly involving salivary and lacrimal glands. It is often overlapped with dermatomyositis. The symptoms are mainly dry mouth and eyes, when it involves the respiratory system, and it can cause severe dry cough or sticky cough. Dysphagia occurs when the digestive system is involved [22, 23]. Commonly used drugs for treatment are prednisone, cyclophosphamide, azathioprine, coenzyme Q10 and vitamins.

Thrombocytopenia in dermatomyositis is associated with the presence of anticardiolipin antibody syndrome. It is prone to thrombosis, and often involved in peripheral blood vessels, cerebrovascular vessels and blood vessels of the heart, lungs, kidneys, etc., which can be treated with immunosuppressants (cyclophosphamide), hormones, heparin, warfarin and aspirin $[24,25]$.

The experience of this paper is summarized as follows: 1) after the diagnosis of dermatomyositis is confirmed, good compliance can be obtained, which is helpful to cure; 2) massive pericardial effusions may cause respiratory and circulatory failure, and it is necessary to promptly convince patients to perform pericardial puncture, anddraineffusion properly; 3) complications such as pulmonary hypertension, dysphagia and SS need to be detected in time and treated actively. Especially when dysphagia occurs, the timely addition of sufficient amount of IVIG is the key to treatment; 4) it is puzzling that the symptoms of the patient suddenly aggravate after hot bath, is it related to low capacity?

In summary, we share the treatment experience of dermatomyositis followed by complications such as massive pericardial effusion, providing case accumulation for the treatment of this disease. A flow chart is used to show the treatment process, which is easy to read and interesting to the reader. There are many deficiencies in this case. For patients with advanced pulmonary hypertension, dysphagia, SS, hyperthermia and anticardiolipin antibody syndrome in the late stage, this article elaborates not enough and there are still many puzzles. Whether the treatment method is appropriate needs further research.

\section{Acknowledgments}

We are grateful to the medical staff who have treated this pa- tient carefully.

\section{Conflict of Interest}

On behalf of all authors, the corresponding author states that there is no conflict of interest. The work has not been published previously, and not under consideration for publication elsewhere, in whole or in part.

\section{Ethical Approval}

This is a clinical observation article. According to the local law, no ethical approval was needed for such an investigation.

\section{References}

1. Bohan A, Peter JB. Polymyositis and dermatomyositis (first of two parts). N Engl J Med. 1975;292(7):344-347.

2. Sigurgeirsson B, Lindelof B, Edhag O, Allander E. Risk of cancer in patients with dermatomyositis or polymyositis. A population-based study. N Engl J Med. 1992;326(6):363-367.

3. Callen JP. Dermatomyositis. Lancet. 2000;355(9197):5357.

4. Kamath S, Roobottom CA. Hyperdense pericardial effusion in dermatomyositis and contrast induced nephropathy. Emerg Radiol. 2005;11(3):177-179.

5. Dalakas MC, Illa I, Dambrosia JM, Soueidan SA, Stein DP, Otero C, Dinsmore ST, et al. A controlled trial of highdose intravenous immune globulin infusions as treatment for dermatomyositis. N Engl J Med. 1993;329(27):19932000.

6. Miossec P. Progress in the treatment of juvenile dermatomyositis. Lancet. 2016;387(10019):627-628.

7. Galimberti F, Kooistra L, Li Y, Chatterjee S, Fernandez AP. Intravenous immunoglobulin is an effective treatment for refractory cutaneous dermatomyositis. Clin Exp Dermatol. 2018;43(8):906-912.

8. Guerra F, Gelardi C, Capucci A, Gabrielli A, Danieli MG. Subclinical Cardiac Dysfunction in Polymyositis and Dermatomyositis: A Speckle-tracking Case-control Study. J Rheumatol. 2017;44(6):815-821.

9. Vakamudi S, Ho N, Cremer PC. Pericardial Effusions: Causes, Diagnosis, and Management. Prog Cardiovasc Dis. 2017;59(4):380-388.

10. Napp LC, Conzen U, Treptau J, et al. Giant pericardial effusion: drain it all? European Heart Journal. 2016;37(29):ehw162.

11. Tanizaki S, Nishida S, Maeda S, Ishida H. Non-surgical management in hemodynamically unstable blunt traumatic pericardial effusion: A feasible option for treatment. Am J Emerg Med. 2018;36(9):1655-1658.

12. Paez M, Wolfert M, Tsang R, et al. Dermatomyositis: A rare but deadly cause of dysphagia. American Journal of Gastroenterology. 2013;108:S199. 
13. Van Mol, Pierre Noreillie, et al. Dysphagia during glucocorticoid treatment of dermatomyositis: a differential diagnostic challenge. Rheumatology Advances in Practice. 2018.

14. de Merieux P, Verity MA, Clements PJ, Paulus HE. Esophageal abnormalities and dysphagia in polymyositis and dermatomyositis. Arthritis Rheum. 1983;26(8):961968.

15. Ertekin C, Secil Y, Yuceyar N, Aydogdu I. Oropharyngeal dysphagia in polymyositis/dermatomyositis. Clin Neurol Neurosurg. 2004;107(1):32-37.

16. Kwon KM, Lee JS, Kim YH. A case report of lifethreatening acute dysphagia in dermatomyositis: Challenges in diagnosis and treatment. Medicine (Baltimore). 2018;97(17):e0508.

17. Joshi D, Mahmood R, Williams P, Kitchen P. Dysphagia secondary to dermatomyositis treated successfully with intravenous immunoglobulin: a case report. Int Arch Med. 2008;1(1):12.

18. Minai OA. Pulmonary hypertension in polymyositisdermatomyositis: clinical and hemodynamic characteristics and response to vasoactive therapy. Lupus. 2009; 18(11):1006-1010.
19. Kato M, Okada T, Kimura R, et al. A difficult case of pulmonary hypertension caused by dermatomyositis. Journal of Cardiac Failure. 2015;21(10):S200-S201.

20. Hoeper MM, Humbert M, Souza R, Idrees M, Kawut SM, Sliwa-Hahnle K, Jing ZC, et al. A global view of pulmonary hypertension. Lancet Respir Med. 2016;4(4):306322.

21. Cottin V. Idiopathic interstitial pneumonias with connective tissue diseases features: A review. Respirology. 2016;21(2):245-258

22. Voulgarelis M, Tzioufas AG. Pathogenetic mechanisms in the initiation and perpetuation of Sjogren's syndrome. Nat Rev Rheumatol. 2010;6(9):529-537.

23. Saito S, Togashi A, Kaneko F, Yamamoto T, Uchida T, Oyama N. Primary Sjogren's syndrome with myalgia mimicking dermatomyositis. J Dermatol. 2010;37(9):837-839.

24. Galli M, Comfurius P, Maassen C, Hemker HC, de Baets $\mathrm{MH}$, van Breda-Vriesman PJ, Barbui T, et al. Anticardiolipin antibodies (ACA) directed not to cardiolipin but to a plasma protein cofactor. Lancet. 1990;335(8705):15441547.

25. Lockshin MD. Anticardiolipin antibody. Arthritis Rheum. 1987;30(4):471-472. 